\title{
Epidémiologie de la rage et connaissance, attitudes et pratiques des communautés au Burkina Faso
}

\author{
Madi Savadogo ${ }^{1,2,3 *}$ Philippe Koné ${ }^{1 \dagger}$ \\ Laibané Dieudonné Dahourou ${ }^{4}$ Rosine Manishimwe ${ }^{5}$ \\ Adama Sow ${ }^{1}$ Lalé Nébié ${ }^{6}$ Nicolas Antoine-Moussiaux ${ }^{2}$ \\ Bernard Doulkom ${ }^{7}$ Rianatou Bada-Alambedji ${ }^{1}$
}

\begin{abstract}
Mots-clés morsure, zoonose, attitude,

Submitted: 27 February 2019

Accepted: 26 March 2020

Published: 11 May 2020

DOI: $10.19182 /$ remvt.31863
\end{abstract}

Chien, animal carnivore, rage, population urbaine, Burkina Faso

\begin{abstract}
Résumé
La rage est une zoonose endémique dans les pays en développement et compte parmi les pathologies virales les plus virulentes et mortelles. Elle constitue une menace pour la santé publique et animale au Burkina Faso. La présente étude transversale a été conduite pour décrire l'épidémiologie de la rage et les connaissances, attitudes et pratiques (CAP) des populations à Ouagadougou. Elle a consisté en une collecte de données auprès des ménages et des structures techniques impliquées dans la prévention, le contrôle et la surveillance de la rage humaine et animale. L'enquête CAP a été réalisée auprès de 616 ménages. Elle a montré que la majorité des participants avaient entendu parler de la rage humaine $(80,7 \%)$ et animale $(94,6 \%)$, savaient qu'elle se transmettait essentiellement à la suite de morsure par un chien infecté $(94,2 \%)$ et étaient capables de citer correctement au moins deux voies de transmission de la maladie $(65,7 \%)$. En revanche, seuls 9,7\% des propriétaires de chiens affirmaient être capables de financer la vaccination antirabique canine annuelle. Les principaux canaux d'information et de communication sur la rage utilisés par les participants étaient l'entourage (41,8\%), l'école $(33,4 \%)$ et les médias $(24,8 \%)$. Quant à l'étude épidémiologique rétrospective, qui s'est appuyée sur les documents disponibles au sein des structures nationales, elle a mis en évidence qu'en moyenne 4172 cas de morsures étaient enregistrés chaque année, que seules $31,7 \%$ des personnes mordues avaient bénéficié d'une prophylaxie postexposition complète et que $68 \%$ des animaux testés étaient positifs au virus rabique. Ces études confirment la présence du risque rabique dans le pays et montrent la nécessité de renforcer l'éducation et l'engagement communautaire ainsi qu'une étroite collaboration entre les secteurs de la santé animale et de la santé humaine pour une gestion intégrée des cas de morsures.
\end{abstract}

- Comment citer cet article: Savadogo M., Koné P., Dahourou L.D., Manishimwe R., Sow A., Nébié L. Antoine-Moussiaux N., et al., 2020. Rabies epidemiology and community knowledge, attitudes and practices in Burkina Faso. Rev. Elev. Med. Vet. Pays Trop., 73 (2): 133-140, doi: 10.19182/remvt.31863

\footnotetext{
1. Ecole inter-Etats des sciences et médecine vétérinaires, BP 5077, Dakar Fann, Sénégal.

2. Institut vétérinaire tropical, Faculté de médecine vétérinaire de l'Université de Liège, Belgique.

3. Institut de recherche en sciences de la santé (IRSS/CNRST), Bobo Dioulasso, Burkina Faso.

4. Institut des sciences de l'environnement et du développement Rurale (ISEDR), Université de Dédougou, Burkina Faso.

5. School of Animal Sciences and Veterinary Medicine, University of Rwanda, Nyagatare, Rwanda.

6. Veterinary Office, Ouagadougou, Burkina Faso.

7. Direction générale des services vétérinaires, Ouagadougou, Burkina Faso.

† Décédé avant la parution du présent manuscrit

* Auteur pour la correspondance

Email : savadogo.madi@yahoo.fr
}

\section{INTRODUCTION}

La rage est une maladie infectieuse due à un virus neurotrope appartenant au genre Lyssavirus (Sabeta et al., 2003). C'est une zoonose majeure, endémique dans les pays en développement. Elle compte parmi les maladies infectieuses les plus mortelles communes à l'homme et aux animaux. La stratégie de prévention et de contrôle de la rage est basée sur la vaccination des carnivores, la prophylaxie postexposition (PPE), la surveillance épidémiologique et l'information des populations. Malgré l'existence d'un vaccin et d'une PPE efficaces, la rage continue de causer des décès dans les pays touchés. A l'échelle du monde, les estimations de mortalité humaine due à la rage s’établissent à 59000 décès par an (Hampson et al., 2015). En Afrique, le virus de la rage représente une menace constante pour les populations puisqu'une personne en meurt toutes les vingt minutes (Dodet et al., 2008). 
Au Burkina Faso, soixante cas humains, dont $40 \%$ d'enfants de moins de quinze ans, ont été enregistrés dans la capitale, Ouagadougou, sur la période de 2003 à 2014 (Kaboré, 2014 ; Sondo et al., 2015). Le nombre annuel de cas de morsures enregistrés est l'un des plus élevés de la sous-région (Sondo et al., 2018 ; Tiembré et al., 2009 ; Diop et al., 2007 ; Dao et al., 2006). Depuis trois décennies, la rage est une maladie légalement réputée transmissible et à déclaration obligatoire sur l'ensemble du pays (Burkina Faso, 1989). Les textes nationaux ont interdit la divagation des carnivores domestiques en zone infectée et rendu obligatoire la vaccination antirabique annuelle des chiens, des chats et des primates en captivité dès l'âge de trois mois sur tout le pays. Tout propriétaire de chien mordeur non vacciné a l'obligation d'assumer les coûts liés à la prise en charge de la personne mordue. Toutefois, d'une manière générale, on note une défaillance dans l'application des textes sur le contrôle et la surveillance de la rage. Par ailleurs, il n'y a pas de législation qui encadre la collaboration intersectorielle et en particulier la gestion intégrée des cas de morsures par les services vétérinaires et les services en charge de la santé humaine.

La PPE est assurée par le Centre national de traitement antirabique (CNTAR), présent uniquement dans les deux principales villes du pays (Ouagadougou et Bobo-Dioulasso). La dose de vaccin y coûtait 13 euros en 2014. Une dose vaccinale dite préventive est administrée aux personnes exposées à la première consultation, en attendant les résultats de l'évaluation du risque de contamination. Le protocole de la PPE n'est complété que pour les patients chez qui la morsure est par la suite jugée contaminante. Le Laboratoire national d'élevage (LNE), localisé à Ouagadougou, réalise le diagnostic expérimental de la rage. Quant aux cliniques vétérinaires, dont celle de l'Ecole nationale d'élevage et de santé animale (ENESA) à Ouagadougou, elles sont en charge de la vaccination canine et de la mise en observation vétérinaire des animaux mordeurs.

Les prestations comme la PPE, le diagnostic expérimental, la mise en observation vétérinaire et la vaccination canine sont financées par les populations. En outre, la plupart des prestations et les vaccins pour humains et animaux ne sont accessibles qu'à Ouagadougou et dans quelques autres grandes villes du pays. La vaccination canine réalisée par les vétérinaires privés coûte en moyenne 15 euros $(€)$. Cette situation rend ces services peu accessibles géographiquement et financièrement pour les populations (Savadogo et Boushan, 2015). Enfin et malheureusement, il n'y a pas de campagnes de vaccination de masse des carnivores domestiques régulièrement organisées. Une telle campagne avait été effectuée en 1999 et avait concerné toutes les provinces du pays. Mais celles organisées ensuite, en 2007, 2011 et 2016, s'étaient limitées le plus souvent à la capitale. La présente étude a été mise en place pour décrire, d'une part, l'épidémiologie de la rage et, d'autre part, les connaissances et les comportements des populations vis-à-vis du risque de la maladie.

\section{MATERIEL ET METHODES}

L'étude a été conduite à Ouagadougou, la plus grande agglomération du Burkina Faso, qui comptait près de 1,5 million d'habitants en 2006 (Burkina Faso, 2009). Elle a consisté en deux enquêtes. La première était une enquête transversale descriptive portant sur la gestion des cas de morsures, le diagnostic du virus et le suivi des animaux mordeurs de janvier 2001 à décembre 2013. La seconde, menée d'août à décembre 2014, a permis la collecte de données sur les connaissances, les attitudes et les pratiques (CAP) des ménages à l'égard de la rage et de la possession de carnivores.

Les données sur les cas de morsures et la mise en œuvre de la PPE de 2001 à 2013 ont été collectées au CNTAR. La PPE y était mise en œuvre selon le protocole d'Essen (Sondo et al., 2014). En ce qui concerne le diagnostic expérimental portant sur les prélèvements de cerveaux d'animaux mordeurs abattus, les données concernant la même période ont été collectées au LNE. La technique utilisée était l'immunofluorescence directe dont le principe consiste à révéler la présence d'antigènes viraux grâce aux anticorps spécifiques précouplés à l'isothiocyanate de fluorescéine (Dean et Abelseth, 1973). A la clinique de l'ENESA, les données portant sur la mise en observation des animaux mordeurs ont été collectées pour la période de 2010 à 2014. Durant cette période de mise en observation de quinze jours, les trois visites sanitaires prévues se faisaient au domicile du propriétaire.

L'enquête sur les connaissances, attitudes et pratiques a été conduite auprès de 616 ménages répartis dans 12 secteurs aléatoirement sélectionnés parmi les 55 que compte la ville. Dans les secteurs concernés, seuls les ménages situés le long des rues principales ont été retenus selon le principe suivant : de part et d'autre d'une rue prise au hasard, chaque troisième ménage a été visité et interrogé. Dans les habitations collectives (ou cours communes) dans lesquelles vivaient plusieurs ménages, une seule personne était interrogée. Les immeubles à usage d'habitation sont assez rares à Ouagadougou, et aucun n'a été inclus dans l'échantillon. Les interviews étaient menées auprès des chefs de ménage ou, à défaut, auprès d'une autre personne vivant dans le ménage et disposée à participer à l'étude. Ces personnes ont été classées dans trois groupes, assimilés à leur statut socioéconomique supposé, en fonction de la somme qu'elles se disaient prêtes (question ouverte) à dépenser pour la vaccination antirabique canine annuelle : faible (moins de $1,5 €$ ), moyen (entre 1,5€ et 7,6 $€$ ), élevé (plus de $7,6 €$ ). Les informations sur les caractéristiques sociodémographiques (sexe, âge, profession, résidence, niveau d'études, taille du ménage, nombre de chiens dans le ménage), les connaissances sur la rage (animaux vecteurs, modes de transmission, mesures préventives, etc.) et la conduite à tenir en cas de morsure ont été collectées. Les données ont été analysées avec le logiciel R 3.1.2 au niveau de confiance de $95 \%$. Le test du chi carré a été utilisé pour étudier l'influence des variables indépendantes (sexe, âge, niveau d'études, statut socioéconomique, type d'habitation et présence de chiens dans le ménage) sur les connaissances et les comportements des personnes. La différence était significative lorsque la valeur de p se situait en dessous de $5 \%$.

\section{RESULTATS}

\section{Données sur les cas de morsures et la prophylaxie postexposition}

Le CNTAR a enregistré 54238 cas de morsures sur la période de l'étude, soit une moyenne de $4172 \pm 829$ personnes mordues chaque année. Cependant, seules $31,7 \%$ (soit 17212 ) d'entre elles ont complété la PPE. Au moment de l'étude, le coût de la PPE était de $13 €$ par dose et le protocole vaccinal d'Essen était utilisé. Au total 54993 doses de vaccins ont été administrées dont le coût actualisé au taux d'inflation de $3 \%$ a été évalué à $85700 €$, représentant ainsi les frais consentis par les personnes pour bénéficier de la PPE. Les frais de traitement étaient à la charge du propriétaire de l'animal mordeur ou, à défaut, de la personne mordue. Les cas de morsures et la mise en œuvre de la PPE ont connu une croissance de 2001 à 2013 (figures 1 et 2). De légères chutes ponctuelles du nombre de personnes mordues ont été observées en 2007 et 2011, années précédées par des campagnes d'abattage de chiens errants dans la ville.

\section{Diagnostic de la rage chez les animaux mordeurs}

Sur la période de 2001 à 2013, le LNE a reçu 3798 échantillons issus de diverses espèces animales. Ces animaux étaient responsables de 4461 morsures et ont été abattus par les populations. Les échantillons provenaient essentiellement de Ouagadougou (87,8\%) et ont été analysés dès leur arrivée au laboratoire. Les résultats ont montré que 68,2 \% 


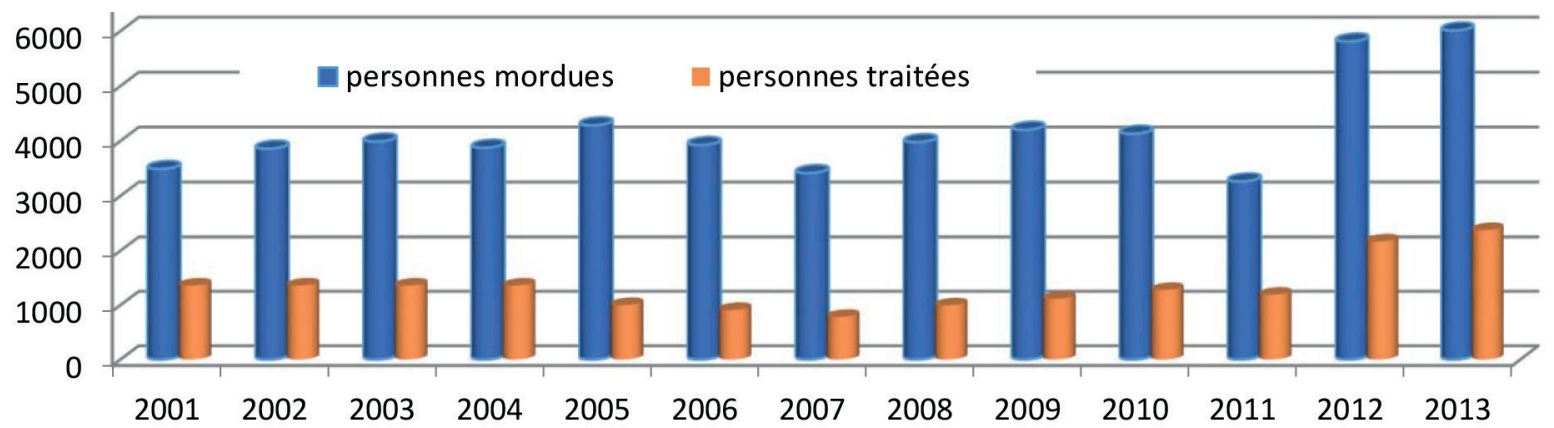

Figure 1 : évolution du nombre de morsures et de personnes traitées contre le virus de la rage à Ouagadougou de 2001 à 2013.

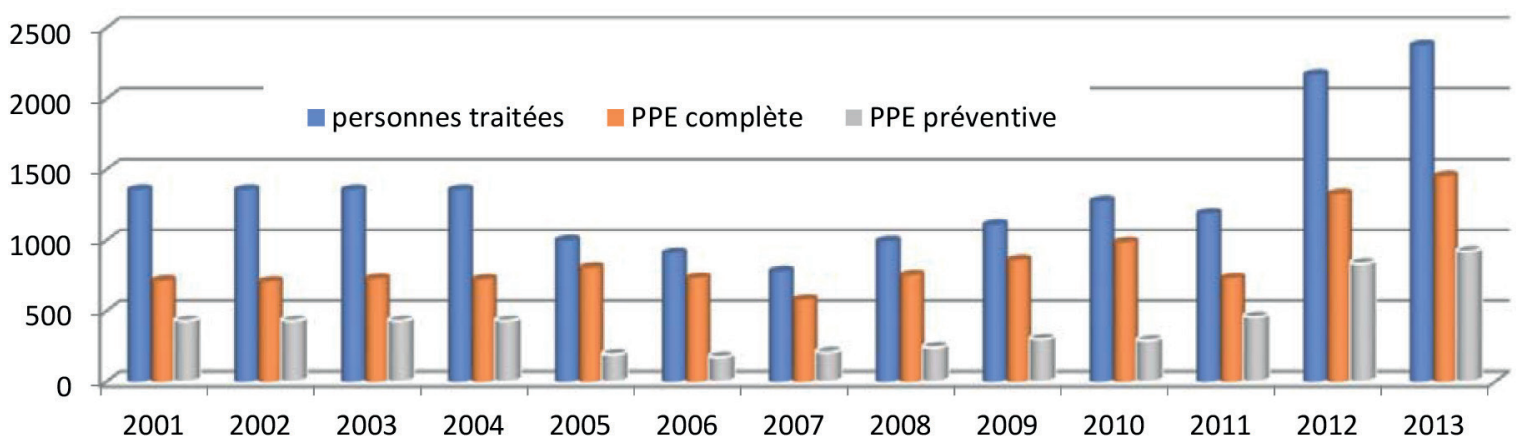

Figure 2 : évolution du nombre de prophylaxies postexposition préventives (une seule injection dans l'attente du résultat de l'examen de l'animal mordeur) ou complètes, administrées aux personnes exposées au virus de la rage à Ouagadougou de 2001 à 2013.

des échantillons étaient positifs à la rage (tableau I). Les espèces animales les plus impliquées dans les cas de morsures étaient les chiens $(90,3 \%)$ et les chats $(7,4 \%)$ avec des taux de positivité à la rage qui étaient respectivement de 70,0\% et 56,8\% (tableau I). En outre, les

\section{Tableau I}

Résultats du diagnostic de rage effectué sur les animaux mordeurs au Laboratoire national d'élevage au Burkina Faso entre 2001 et 2013

\begin{tabular}{lrcc} 
Espèce animale & Nombre & Positif $\mathbf{\%})$ & $\begin{array}{c}\text { Intervalle de } \\
\text { confiance }\end{array}$ \\
\hline Chien & 3431 & 70,0 & $68,4-71,5$ \\
Chat & 280 & 56,8 & $50,9-62,5$ \\
Singe & 24 & 41,7 & $24,5-61,2$ \\
Souris & 18 & 22,2 & $3,0-41,4$ \\
Rat & 8 & 50,0 & - \\
Musaraigne & 12 & 41,6 & $13,7-69,5$ \\
Lagomorphe & 5 & 0 & - \\
Cheval & 1 & 0 & - \\
Ane & 8 & 37,5 & - \\
Bovin & 1 & 0 & - \\
Ovin & 2 & 0 & - \\
Caprin & 3 & 33,3 & - \\
Chauve-souris & 1 & 100,0 & - \\
Chacal & 2 & 50,0 & - \\
Dromadaire & 1 & 100,0 & - \\
Porc & 1 & 0 & - \\
Total & 3798 & 68,2 & $66,7-69,7$
\end{tabular}

résultats ont été positifs chez $34,2 \%$ des 38 micromammifères examinés $(22,2 \%$ chez les souris, $50 \%$ chez les rats et $41,6 \%$ chez les musaraignes). La figure 3 montre que le taux de positifs chez les différentes espèces animales a connu une nette progression de 2001 à 2013. Seuls $13 \%$ des 3431 chiens mordeurs prélevés étaient correctement vaccinés, selon les indications fournies par le registre du LNE.

\section{Suivi sanitaire des animaux mordeurs}

De 2010 à 2013, 5881 animaux mordeurs ont été mis en observation à la clinique de l'ENESA. Ils appartenaient à trois espèces animales : le chien en grande majorité $(93,5 \%)$, le chat $(6,4 \%)$ et le singe $(0,1 \%)$. En moyenne, $1371 \pm 345$ chiens ayant mordu des personnes étaient reçus chaque année à la clinique pour le suivi. Ces chiens mordeurs avaient un propriétaire et étaient accessibles et maîtrisables. La mise en observation était réalisée par le vétérinaire, l'animal étant gardé au domicile du propriétaire pendant les 15 jours requis. L'absence de données sur le devenir des animaux mordeurs mis en observation n'a pas permis d'établir de liens entre ces animaux et ceux dont les prélèvements ont été testés au laboratoire.

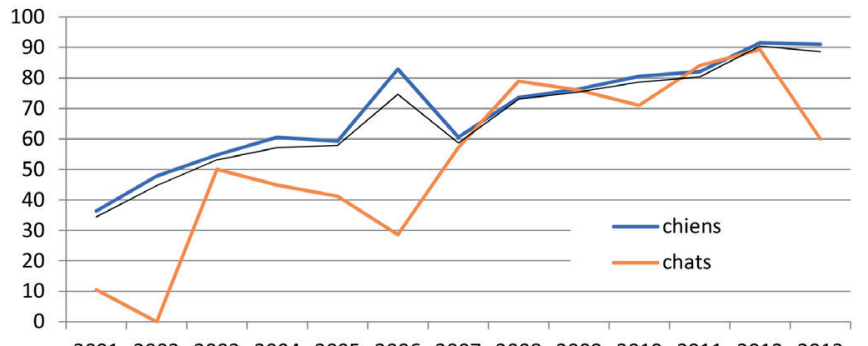

2001200220032004200520062007200820092010201120122013

Figure 3: évolution du taux de positivité chez les animaux testés au laboratoire de 2001 à 2013. 


\section{Connaissances sur la rage et comportements des personnes à l'égard des mesures de lutte}

L'âge moyen des 616 personnes enquêtées était de 33 ans, 63,8\% d'entre elles avaient au moins un niveau d'études secondaires et 57,3\% était des hommes. L'enquête a permis de dénombrer 461 chiens et 3848 personnes dans les 616 ménages visités, dont 57,6\% avaient au moins un chien. Le ratio humain/chien calculé à partir de ces informations a donné la valeur de 8,3 personnes pour un chien. Le chien était essentiellement utilisé pour la garde $(64,7 \%)$. L'étude a révélé que 33,5\% des propriétaires avaient leurs chiens correctement vaccinés.

La plupart des personnes avaient déjà entendu parler de la rage humaine $(80,7 \%)$ et animale $(94,6 \%)$. Le chien, le chat et le singe ont été les animaux les plus cités pouvant transmettre la rage par morsure ou griffure. Les autres espèces animales citées étaient les rongeurs, les ruminants, les équidés, les reptiles et les chauves-souris. Concernant la prévention de la rage animale, $88,3 \%$ des répondants ont mentionné la vaccination antirabique et le confinement comme mesures efficaces, alors que la majorité des propriétaires de carnivores domestiques ne connaissaient ni l'âge de la primovaccination $(78,9 \%)$ ni la fréquence des rappels vaccinaux $(59,4 \%)$. Les personnes qui avaient un niveau d'études secondaire ou universitaire ainsi que celles qui avaient un chien avaient une meilleure connaissance de la rage $(\mathrm{p}<0,05)$ (tableau II). Seuls 9,7\% des propriétaires, de statut socioéconomique supposé élevé car faisant partie des 13,5\% disposés à dépenser plus de 7,6 euros annuellement pour la vaccination, se disaient capables d'allouer annuellement 15 euros pour vacciner leur animal. Le statut socioéconomique supposé de la majorité des personnes enquêtées était soit faible $(35,7 \%)$, soit moyen (50,8 \%). A la question de savoir qui devait prendre en charge la vaccination antirabique des carnivores domestiques, les propriétaires ont majoritairement répondu qu'elle devait leur incomber (65,9\%) (tableau II).

La connaissance des responsabilités incombant aux propriétaires de chiens était liée au statut socioéconomique des personnes enquêtées $(\mathrm{p}<0,05)$ (tableau II). Les personnes ayant un statut socioéconomique moyen à élevé étaient respectivement $72,2 \%$ et $74,7 \%$ à considérer que la vaccination du chien devait relever de la responsabilité du propriétaire contre $55,9 \%$ chez les personnes au statut socioéconomique faible. Parmi les propriétaires de chien, 18,0 \% (64/355) ont affirmé que leurs chiens avaient déjà mordu une personne, mais seulement $59,4 \%(38 / 64)$ de ces chiens mordeurs avaient été mis en observation par un vétérinaire. Les résultats relatifs à la conduite à tenir en cas de morsure par un animal suspect ont montré que $67,4 \%$ des personnes savaient qu'il fallait immédiatement consulter un médecin et demander une mise en observation de l'animal mordeur. La maîtrise de la conduite à tenir était associée à l'âge, au sexe, au niveau d'études et à la présence d'un chien dans le ménage $(\mathrm{p}<0,05)$. L'enquête a également révélé que les principaux canaux d'information sur la rage étaient l'entourage (41,8\%), l'école (33,4\%) et les médias $(24,8 \%)$.

Concernant la mise en œuvre effective des mesures de prévention par les populations, les résultats ont montré qu'il existait des logiques sociales et économiques qui influaient sur la prise de décision de vacciner les carnivores domestiques (tableau III). La perception du coût de la vaccination par les propriétaires n'avait pas d'influence significative sur le statut vaccinal des chiens $(p>0,05)$. Néanmoins la plupart des personnes qui n'avaient pas vacciné leurs chiens ont estimé que la vaccination était chère. La distance entre le domicile du propriétaire et la clinique vétérinaire la plus proche, le mode de tenue du chien par le propriétaire (attaché ou divagant), les caractéristiques intrinsèques du chien (race et sexe) avaient une influence significative sur le statut vaccinal $(\mathrm{p}<0,05)$.

\section{DISCUSSION}

L'étude a montré que le virus rabique représentait une véritable menace de santé publique au Burkina Faso. Les données émanaient de structures localisées à Ouagadougou mais leur collection provenait de différentes régions du pays. Néanmoins la quasi-totalité des informations rapportées concernait la zone de Ouagadougou. D'autres études menées en milieux rural et urbain avaient montré que la maladie était inégalement répartie à travers le pays et que les grands centres urbains connaissaient les plus fortes prévalences (Kouldiati, 1989). Est-ce la véritable situation ou une impression due à la méconnaissance ou à la négligence des communautés rurales, ou à l'inaccessibilité des services techniques en charge du contrôle et de la surveillance par ces mêmes communautés ?

En 1989, Kouldiati a observé un cumul de 1687 personnes mordues entre 1981 et 1987 à Ouagadougou, soit 241 par an. Le nombre de cas annuel de morsures recensés dans cette ville entre 2001 et 2013 est bien plus élevé (plus de 4000) que ceux rapportés à Casablanca au Maroc (Institut Pasteur du Maroc, 2014), à Abidjan en Côte d'Ivoire (Tiembré et al., 2009) et à Bamako au Mali (Dao et al., 2006), villes dont la taille de la population est au moins égale à celle de Ouagadougou. Plus de la moitié des personnes mordues n'ont pas complété la PPE, sans explication connue. La question du devenir de ces personnes exposées se pose car $70 \%$ des chiens mordeurs examinés au laboratoire étaient positifs à la rage. Malheureusement, il n'existe aucun système de suivi, d'alerte et de rappel des patients en cours de traitement. Dans ce contexte, plusieurs patients qui ont bénéficié d'une PPE préventive, dans l'attente des conclusions de la mise en observation de l'animal mordeur ou des analyses de laboratoire, ne se sont plus présentés au CNTAR afin de compléter la prophylaxie alors que le diagnostic était positif.

Sur la période de l'étude, la tendance globale a montré une hausse de la quantité de PPE depuis 2001. Malgré les minicampagnes d'abattage de chiens errants réalisées en 2006 et 2010, la baisse du nombre de morsures a été aussi éphémère que légère en 2007 et 2011, avec un nombre plus important de cas de morsures les années suivantes. Plusieurs études ont d'ailleurs montré qu'une approche basée sur l'abattage des chiens est inefficace, voire contre-productive à long terme, et n'a qu'un effet temporaire sur la population canine et l'incidence de la maladie (Moreira et al., 2004 ; Wera et al., 2013 ; Cleaveland et al., 2014 ; Yaok et al., 2016).

L'augmentation du nombre de morsures notifiées durant les deux dernières années de l'étude (2012 et 2013) s'expliquerait par une meilleure prise de conscience de la gravité de la maladie qui amènerait les victimes à consulter les services de santé. Ce constat révèle l'importance de l'information et de l'éducation communautaire dans la prévention et le contrôle de la rage (Sambo et al., 2014 ; Mosimann et al., 2017). Comme rapporté par Jackson (2013) et Mindekem (2017), les données recueillies montrent que le chien est le principal animal responsable des cas de morsures des personnes.

Le nombre annuel de mises en observation d'animaux mordeurs était largement inférieur à celui de personnes mordues. Cette situation traduit l'absence de collaboration étroite entre les acteurs de la santé humaine et de la santé animale, ce qui est comparable à la situation décrite à Ndjamena au Tchad (Mindekem, 2017). En raison de la faible collaboration entre les médecins et les vétérinaires, les médecins du CNTAR qui reçoivent les personnes mordues procèdent souvent à l'administration de la PPE, sans exiger systématiquement une mise en observation de l'animal mordeur ou un test de laboratoire sur le mordeur mort, pourtant nécessaire à l'évaluation du risque de contamination. Le manque de communication entre les acteurs se traduit également par l'absence de données sur le devenir des animaux mordeurs mis en observation, d'où les difficultés à établir le 


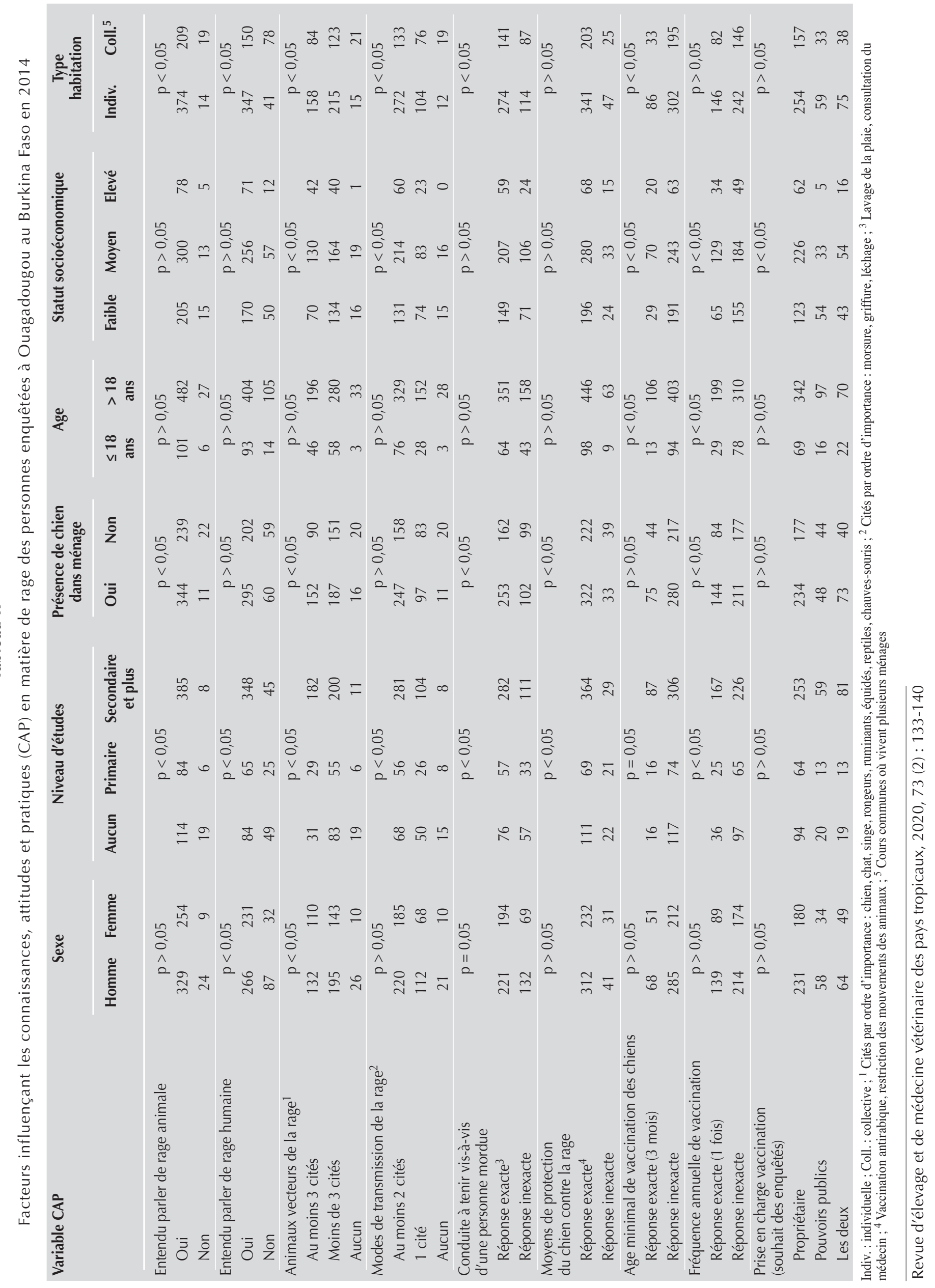




\section{Tableau III}

Influences socioéconomiques sur la décision de faire vacciner son chien contre la rage à Ouagadougou au Burkina Faso en $2014(\mathrm{n}=355)$

Variables$$
\text { . }
$$

Appréciation du coût
de la vaccination
Abordable
Cher

Eloignement estimé domicile du propriétaire - clinique vétérinaire

$<5 \mathrm{~km}$
$\geq 5 \mathrm{~km}$

Mode de tenue du chien

En confinement $24 \mathrm{~h} / 24$

En liberté

Sexe du chien

Mâle

Femelle

Race du chien

Race commune ou locale

Race exotique

\section{Statut vaccinal Vacciné Non Inconnu}

$p>0,05$

46
73

24

73

50

89

$\mathrm{p}<0,05$

$\begin{array}{lll}91 & 31 & 68\end{array}$

28

43

$\mathrm{p}<0,05$

23 49

$\begin{array}{lcc}82 & 23 & 49 \\ 37 & 51 & 113\end{array}$

$\mathrm{p}<0,05$

$\begin{array}{lll}73 & 67 & 72\end{array}$

$46 \quad 7$

$\mathrm{p}<0,05$

$90 \quad 74$

$29 \quad 0$

162

0

lien entre ces animaux suivis et les prélèvements testés au laboratoire. Cette situation peut s'expliquer en partie par le contexte prévalant au Burkina Faso où de nombreux chiens considérés comme domestiques sont en réalité des chiens divagants ou errants occasionnels ayant un propriétaire. Par son utilité et ses multiples fonctions dans la société burkinabè, le chien est l'animal de compagnie le plus détenu par les ménages. Ces chiens, souvent laissés en liberté et mal nourris par les propriétaires, se promènent dans les rues et les décharges pour s'alimenter. Cette situation rend difficile le suivi des chiens mordeurs d'autant plus qu'il se fait généralement au domicile du propriétaire, à condition que ce dernier soit identifié et le chien maîtrisable. Par ailleurs, les animaux étant souvent en liberté, il arrive que des chiens mordeurs correctement vaccinés soient abattus par les populations, ce qui peut, à long terme, contribuer à décourager les propriétaires à vacciner.

Les taux de positivité ont été plus élevés chez l'espèce canine, ce qui impliquait que les morsures étaient contaminantes. Des études similaires ont observé des taux de positivité encore plus élevés chez le chien au Mali et en Centrafrique où ils étaient respectivement de $96 \%$ (Dao et al., 2006) et 94,2\% (Mangué, 2009). Il se peut que le taux de positivité obtenu à Ouagadougou ait été sous-estimé puisque tous les animaux morts ne sont pas signalés par les populations, comme c'est le cas dans certains pays de la sous-région, par exemple au Sénégal (Migan, 2007; Nodjimadji, 2008) et en Côte d'Ivoire (Selly Essis et al., 1992). L'éloignement des populations rend difficile l'acheminement des prélèvements vers le laboratoire situé à Ouagadougou, ce qui explique que l'essentiel des prélèvements examinés provenait de la capitale.

La forte positivité de la rage observée chez les chiens examinés peut aussi s'expliquer par la faible couverture vaccinale des carnivores (Savadogo, 2015). On peut s'interroger sur les principales raisons de la non-vaccination des chiens à Ouagadougou, le vaccin étant disponible dans les cliniques vétérinaires localisées dans différentes zones de la ville. Le contexte du Burkina est marqué par la rareté des campagnes de vaccination de masse aux prix subventionnés. Or, on sait que dans les pays endémiques, la vaccination antirabique se heurte souvent à la faible capacité financière des propriétaires de chiens (Lembo et al., 2010).

L'enquête sur les connaissances, attitudes et pratiques en matière de rage présente des informations collectées auprès des chefs de ménages visités ou de leurs représentants. L'étude informe sur certaines logiques sociales qui peuvent affecter l'efficacité des interventions pour la prévention et le contrôle de la rage au Burkina Faso. La plupart des personnes interrogées avaient fait au moins des études secondaires, ce qui s'expliquerait par le fait que l'étude a été réalisée en milieu urbain. Or, le niveau d'instruction des populations influe considérablement sur les perceptions et les attitudes qu'elles ont sur les questions de santé publique (HCSP France, 1998). La quasi-totalité des personnes interrogées avait déjà entendu parler de la rage animale et humaine. Des résultats similaires avaient été trouvés en Tanzanie (Sambo et al., 2014), au Sénégal (Adjé et al., 2015) et au Tchad (Mindekem et al., 2005). Cependant, à l'opposé des constats faits par Adjé et al. en milieu estudiantin (2015), notre étude a montré que l'entourage était le principal canal d'information de la population. Il paraît donc nécessaire d'intensifier l'information et l'éducation communautaire pour une meilleure prise en compte du risque rabique dans les ménages. L'utilisation des posters et des crieurs publics a ainsi révélé l'effet potentiel de la communication dans l'amélioration de la couverture vaccinale des chiens au Mali (Mosimann et al., 2017). Des campagnes médiatiques et, de plus en plus, l'utilisation des réseaux sociaux peuvent offrir de plateformes efficaces pour une large information des communautés sur la maladie et les moyens de prévention.

Les personnes qui avaient un niveau d'études au moins du secondaire et celles qui vivaient dans des ménages possédant un chien connaissaient mieux les animaux vecteurs, les modes de transmission et la conduite à tenir en cas de morsure par un chien. L'effet de l'éducation sur la connaissance de la maladie indiquait par ailleurs que le risque rabique était plus important dans les franges les plus vulnérables de la société où l'accès à l'éducation formelle restait difficile. Ces observations confirment les résultats obtenus par Ahme et al. en 2014.

Concernant la vaccination des animaux par les propriétaires, nos résultats sont similaires à ceux obtenus en Côte d'Ivoire (Tiembré et al., 2009) selon lesquels les personnes interrogées considèrent la vaccination antirabique des chiens comme étant le meilleur moyen de lutter contre la rage. En dépit de cette connaissance exprimée par les propriétaires, le taux de couverture vaccinale des chiens demeure faible, probablement, comme cela a été déjà signalé, par manque de moyens financiers, mais peut-être aussi à cause de l'absence d'un programme national de contrôle de la rage. Dans certaines parties du monde, de tels programmes incluant des campagnes de vaccination de masse ont permis d'éliminer la rage (Bögel et Meslin, 1990 ; Zinsstag et al., 2017).

Concernant la conduite à tenir vis-à-vis du risque rabique, l'étude a montré qu'au moins la moitié des animaux mordeurs ayant un propriétaire étaient mis en observation, taux supérieur à celui de 4,3\% relevé au Maroc (Institut Pasteur du Maroc, 2014). Cependant, de manière générale, les pratiques des populations étaient moins satisfaisantes que celles décrites en Tanzanie (Sambo et al., 2014), bien que les connaissances sur la maladie et les mesures de prévention étaient relativement élevées. Se pose alors la question de comprendre les logiques des populations qui peuvent expliquer l'écart entre leurs connaissances et leurs comportements. Leétude a montré que le prix de la vaccination n'a apparemment pas eu d'influence significative sur la décision des propriétaires à vacciner leurs chiens. Cependant, la faiblesse du pouvoir d'achat pourrait favoriser une faible accessibilité 
financière à la vaccination (Savadogo et Boushan, 2015 ; Lembo et al., 2010 ; Sondo et al., 2018). Par ailleurs, les distances assez longues entre les domiciles des propriétaires et les cliniques vétérinaires provoquent des coûts indirects liés au frais de déplacement et à la perte de temps de travail. Nos observations confortent les travaux de Mosimann et al. (2017) qui rapportent qu'à Bamako, au Mali, le manque de temps des propriétaires et les difficultés à conduire le chien au site de vaccination sont des obstacles à la vaccination. Il y a également des obstacles culturels liés au transport des chiens, certaines personnes ne voulant pas montrer publiquement leur attachement à un chien. La divagation des animaux est fréquente et de nombreuses personnes ont des chiens qui sont laissés en liberté et se nourrissent hors des domiciles, ce qui pose le problème de leur maîtrise par les propriétaires en vue de la vaccination antirabique. D'autres études montrent que les chiens en semi-liberté ou en liberté, fréquemment absents du domicile et agressifs, sont difficiles d'accès par les propriétaires (WHO, 1992 ; Mosimann et al., 2017). L'intensification des campagnes d'information et la mise en œuvre effective des textes réglementaires en vigueur sont nécessaires afin d'améliorer les comportements des populations.

\section{- CONCLUSION}

L'étude a mis en évidence une circulation du virus rabique chez plusieurs espèces animales à Ouagadougou, le chien étant la première cause de morsures des personnes. Les populations ont une connaissance assez satisfaisante de la maladie et des mesures de prévention, mais des comportements et des pratiques à risque persistent au sein des communautés. Afin de renforcer la prévention et le contrôle de la rage au Burkina Faso, il convient de promouvoir une collaboration transsectorielle basée sur l'approche One Health en vue de renforcer la synergie d'action entre toutes les parties prenantes (professionnels de la santé humaine, de la santé animale et de la gestion de la faune, propriétaires de carnivores domestiques, autorités administratives et locales, société civile, et les communautés). Nous recommandons également la mise en place d'études plus approfondies pour évaluer l'impact socioéconomique de la rage et la qualité du dispositif de contrôle et de surveillance au Burkina Faso.

\section{Remerciements}

Les auteurs remercient les populations de Ouagadougou pour la franche collaboration lors de la collecte des données. L'étude a été réalisée avec l'appui technique et logistique de la Direction générale des services vétérinaires (DGSV) du Burkina Faso. Le financement nécessaire à la réalisation des enquêtes a été apporté par les auteurs.

\section{Contributions des auteurs}

MS, PK et BD ont participé à la conception et à la planification de l'étude ; MS a collecté les données ; MS et LDD ont participé à l'analyse et l'interprétation des données ; MS et PK ont rédigé la première version du manuscrit ; RM, AS, LN, NAM et RBA ont réalisé une révision critique du manuscrit. Tous les auteurs ont lu et approuvé la version finale de l'article.

\section{Conflits d'intérêts}

L'étude a été réalisée sans aucun conflit d’intérêts.

\section{REFERENCES}

Adjé K.J.F., N’Dour A.P.N., Coulibaly F., Halidou A.I., Mabeki R., Koné P., 2015. Knowledge, attitudes, and practices concerning rabies and post-exposure prophylactic measures at the Cheikh Anta Diop University in Dakar. Méd. Mal. Infect., 45 (7): 306-307, doi: 10.1016/j. medmal.2015.05.002
Ahme V.O., Dzikwi A.A., Umoh J.U., 2014. Assessment of knowledge, attitude and practice of dog owners to canine rabies in Wukari Metropolis, Taraba State Nigeria. Glob. J. Health Sci., 6 (5): 226-240, doi: 10.5539/ gjhs.v6n5p226

Bögel K., Meslin F.X., 1990. Aspects économiques de l'élimination de la rage humaine et canine : principes pour l'orientation des programmes. Bull. World Health Organ., 68 (4): 409-418

Burkina Faso, 1989. Kiti $n^{\circ}$ AN VII-0113/FP/AGRI-EL, repris par décret $n^{\circ}$ 2018-0730/PRES/PM/MRAH/MINEFID/MATD/MSECU/MCIA portant réglementation de la police zoo-sanitaire, Ouagadougou, Burkina Faso

Burkina Faso, 2009. Annuaire statistique. Ministère de la Santé, Direction générale de l'information et des statistiques sanitaires, Ouagadougou, Burkina Faso

Cleaveland S., Lankester F., Townsend S., Lembo T., Hampson K., 2014. Rabies control and elimination: a test case for One Health. Vet. Rec., 175 (8): 188-93, doi : 10.1136/vr.g4996

Dao S., Abdillahi A.M., Bougoudogo F., Touré K., Simbé C., 2006. Aspects épidémiologiques de la rage humaine et animale en milieu urbain à Bamako, Mali. Bull. Soc. Pathol. Exot., 99 (3) : 183-186

Dean D.L., Abelseth M.K., 1973. The fluorescent antibody test. In: Laboratory techniques in Rabies, $3^{\text {rd }}$ ed. (Ed. Kaplan M.M., Koprowski H.). WHO, Geneva, Switzerland, 73-84

Diop S.A., Manga N.M., Dia N.M., Ndour C.T., Seydi M., Soumare M., Diop B.M., et al., 2007. Le point sur la rage humaine au Sénégal de 1986 à 2005. Méd. Mal. Infect., 37 (12) : 787-791, doi: 10.1016/j. medmal.2007.01.010

Dodet B., AfroREB, Adjogoua E.V., Aguemon A.R., Amadou O.H., Atipo A.L., Baba B.A., et al., 2008. Fighting rabies in Africa: The Africa Rabies Expert Bureau. Vaccine, 26 (50): 6295-6298, doi: 10.1016/j.vaccine.2008.04.087

Hampson K., Coudeville L., Lembo T., Sambo M., Kieffer A., Attlan M., Barrat J., et al., 2015. Estimating the global burden of endemic canine rabies. PLoS Negl. Trop. Dis. 9 (5): e0003709, doi: 10.1371/journal.pntd.0003709

HCSP France, 1998. La progression de la précarité en France et ses effets sur la santé. ENSP, Rennes, France, 368 p.

Institut Pasteur du Maroc, 2014. La rage, cette maladie difficile à combattre au Maroc. Lettre Pasteur (3) : 1-16

Jackson A.C., 2013. Current and future approaches to the therapy of human rabies. Antiviral Res., 99 (1): 61-67, doi: 10.1016/j.antiviral.2013.01.003

Kaboré B.E., 2014. Place de l'enfant parmi les cas de rage dans la ville de Ouagadougou : étude rétrospective sur 10 ans 6 mois. Thèse Méd. Université de Ouagadougou, Burkina Faso, 89 p.

Kouldiati J., 1989. Contribution à l'étude de la rage canine au Burkina Faso: épidémiologie et prophylaxie. Thèse Méd. Vét., EISMV, Dakar, Sénégal, $121 \mathrm{p}$.

Lembo T., Hampson K., Kaare M.T., Ernest E., Knobel D., Kazwala R.R., Haydon D.T., et al., 2010. The feasibility of canine rabies elimination in Africa: dispelling doubts with data. PloS Negl. Trop. Dis., 4 (2): e626, doi: 10.1371/journal.pntd.0000626

Mangué J.S., 2009. Epidémiologie de la rage et aspects moléculaires du virus rabique à Bangui (République centrafricaine) au cours de la période de 2006 à 2008. Thèse Méd. Vét., EISMV, Dakar, Sénégal, 151 p.

Migan A.G., 2007. Contribution à l'étude de l'épidémiologie de la rage au Sénégal au cours de la période de 1996 à 2005. Thèse Méd. Vét., EISMV, Dakar, Sénégal, 86 p.

Mindekem R., 2017. La rage canine au Tchad : état des lieux et qualité du dispositif de lutte. Thèse Doct., Université d'Abomey-Calavi, Bénin, 172 p.

Mindekem R., Kayali U., Yemadji N., Ndoutamia A.G., Zinsstag J., 2005. La démographie canine et son importance pour la transmission de la rage humaine à Ndjamena. Med. Trop., 65 (1) : 53-58

Moreira, E.D. Jr, Mendes de Souza V.M., Sreenivasan M., Nascimento E.G., Pontes de Carvalho L., 2004. Assessment of an optimized dogculling program in the dynamics of canine Leishmania transmission. Vet. Parasitol., 122 (4): 245-252, doi: 10.1016/j.vetpar.2004.05.019

Mosimann L., Traoré A., Mauti S, Léchenne M., Obrist B., Véron R. Hattendorf J., et al., 2017. A mixed methods approach to assess animal vaccination programmes: the case of rabies control in Bamako, Mali. Acta Trop., 165: 203-215, doi: 10.1016/j.actatropica.2016.10.007 
Nodjimadji R., 2008. Contribution à l'étude de l'épidémiologie de la rage au Sénégal : cas de la région de Fatick au cours de la période de 1998 à 2007. Thèse Méd. Vét., EISMV, Dakar, Sénégal, 135 p

Sabeta C.T., Bingham J., Nel L.H., 2003. Molecular epidemiology of canid rabies in Zimbabwe and South Africa. Virus Res., 91 (2): 203-211, doi: 10.1016/S0168-1702(02)00272-1

Sambo M., Lembo T., Cleaveland S., Ferguson H.M., Sikana L., Simon C. Urassa H., et al., 2014. Knowledge, Attitudes and Practices (KAP) about rabies prevention and control: A community survey in Tanzania. PLoS Negl. Trop. Dis., 8 (12): e3310, doi: 10.1371/journal.pntd.0003310

Savadogo M., 2015. Amélioration de la connaissance de l'épidémiologie de la rage au Burkina Faso : Situation épidémiologique et connaissancesattitudes-perceptions sur la rage dans la commune de Ouagadougou. Thèse Méd. Vét., EISMV, Dakar, Sénégal, 113 p.

Savadogo M, Boushab M.B., 2015. La rage chez I'enfant: un risque encore méconnu des populations exposées. Méd. Santé Trop., 25 : 222-224, doi : 10.1684/mst.2015.0449

Selly Essis A.M., Dadou G., Ehouman A., Edoh V., 1992. Evolution de l'endémie rabique dans les populations humaines et animales en Côte d'ivoire. Méd. Afr. Noire, 39 (2) : 86-93

Sondo K.A., Diallo I., Savadogo M., Kaboré B.E., Ouédraogo G.A., Zoungrana J., Diendéré E.A., et al., 2018. Enquête transversale sur les cas de rage reçus de 2003 à 2014 au Service des maladies infectieuses du centre hospitalier universitaire Yalgado Ouédraogo (Burkina Faso). Sci. Tec. Sci. Vie, 1 (1) : 41-5

\section{Summary}

Savadogo M., Koné P., Dahourou L.D., Manishimwe R., Sow A., Nébié L., Antoine-Moussiaux N., Doulkom B., Bada-Alambedji R. Rabies epidemiology and community knowledge, attitudes and practices in Burkina Faso

Rabies is an endemic zoonosis in developing countries and is one of the most virulent and deadly viral diseases. It is a threat to public and animal health in Burkina Faso. This cross-sectional study describes the epidemiology of rabies and the knowledge, attitudes and practices (KAP) of the populations in Ouagadougou. Data were collected from households and technical services involved in the prevention, control and surveillance of human and animal rabies. The KAP survey was conducted among 616 households. It showed that the majority of participants had heard of human (80.7\%) and animal (94.6\%) rabies, were aware that the disease is mainly transmitted by infected dog bites (94.2\%), and were able to quote at least two routes of rabies transmission ( $65.7 \%)$. In contrast, only $9.7 \%$ of dog owners claimed to be able to afford annual canine rabies vaccination. The main channels of information and communication about rabies used by the participants were friends and family $(41.8 \%)$, school (33.4\%), and media (24.8\%). As for the retrospective epidemiological study, which was based on the documents available within national services, it showed that an average of 4172 cases of bites were recorded each year, that only $31.7 \%$ of those bitten had received complete post-exposure prophylaxis, and that $68 \%$ of the animal tested were positive for rabies virus. These studies confirm the presence of a rabies risk in the country, and show the need for increased education and community involvement, and close collaboration between the animal and human health sectors for integrated bite-case management.

Keywords: dogs, carnivorous animals, rabies, bites, zoonoses, attitudes, urban population, Burkina Faso
Sondo K.A., Yonaba/Okenga C., Diop S.A., Kaboré B.E., Diallo I., Kyelem N., Basshono J., et al., 2015. Rabies in children: report of 24 cases at the Yalgado Ouedraogo University Hospital Center of Ouagadougou in Brukina Faso. J. Trop. Dis., 3: 168, doi: 10.4172/2329-891X.1000168

Sondo K.A., Zougrana J., Bazié K., Kyelem C.G., Diallo I., Korsaga N., Basshono J., et al., 2014. Prise en charge postexposition des victimes d'agression par un animal à Ouagadougou. Sci. Tech. Sci. Santé, 37 (1-2) : $27-36$

Tiembré I., Aka-Kone D.M.B., Konan Y.E., Vroh J.B.B., Ekra K.D., Dagnan N.S., Aka J., et al., 2009. Observance du traitement vaccinal antirabique chez les sujets exposés à la rage à Abidjan (Côte d'Ivoire). Santé Publique, 21 (6) : 595-603, doi: 10.3917/spub.096.0595

Wera E., Velthuis A.G.J., Geong M., Hogeveen H., 2013. Costs of rabies control: An economic calculation method applied to Flores Island. PLoS ONE, 8 (12): e83654, doi: 10.1371/journal.pone.0083654

WHO, 1992. Report of the 3rd consultation on oral immunization of dogs against rabies. WHO, Geneva, Switzerland, $14 \mathrm{p}$.

Yaok A.J., Reece J.F., Gehrt S.D., Hamilton I.M., 2016. Optimizing freeroaming dog control programs using agent-based models. Ecol. Model., 341: 53-61, doi: 10.1016/j.ecolmodel.2016.09.018

Zinsstag J., Lechenne M., Laager M., Mindekem R., Naïssengar S., Oussiguéré A., Bidjeh K., et al., 2017. Vaccination of dogs in an African city interrupts rabies transmission and reduces human exposure. Sci. Transl. Med., 9: eaaf6984, doi: 10.1126/scitranslmed.aaf6984

\section{Resumen}

Savadogo M., Koné P., Dahourou L.D., Manishimwe R., Sow A., Nébié L., Antoine-Moussiaux N., Doulkom B., Bada-Alambedji R. Epidemiología de la rabia y conocimiento, actitudes y prácticas de las comunidades de Burkina Faso

La rabia es una zoonosis endémica en los países en desarrollo y se encuentra entre las patologías virales más virulentas y mortales. Constituye una amenaza para la salud pública y animal en Burkina Faso. El presente estudio transversal, se condujo con el fin de describir la epidemiología de la rabia y los conocimientos, actitudes y prácticas (CAP) de las poblaciones en Uagadugú. Consistió en recolectar datos en los hogares y en las estructuras técnicas implicadas en la prevención, el control y la supervisión de la rabia humana y animal. La encuesta CAP se realizó en 616 hogares. Mostró que la mayoría de los participantes habían oído hablar de la rabia humana $(80,7 \%)$ y animal (94,6\%), sabían que se transmitía esencialmente mediante la mordida de un perro infectado $(94,6 \%)$ y fueron capaces de citar correctamente al menos dos vías de transmisión de la enfermedad (65,7\%). Por otro lado, sólo 9,7\% de los propietarios de perros afirmaron ser capaces de financiar la vacunación anti rábica anual canina. Los principales canales de información y de comunicación sobre la rabia, utilizados por los participantes, fueron el entorno $(41,8 \%)$, la escuela $(33,4 \%)$ y los medios de comunicación (24,8\%). En cuanto al estudio epidemiológico retrospectivo, basado en los documentos disponibles en las estructuras nacionales, puso en evidencia que, en promedio, se registraron 4,172 casos de mordidas cada año, que solamente $31,7 \%$ de las personas mordidas beneficiaron de una profilaxis post exposición completa y que $68 \%$ de las muestras animales examinadas fueron positivas al virus de la rabia. Estos estudios confirman la presencia del riesgo de rabia en el país y muestra la necesidad de reforzar la educación y la responsabilidad comunitaria, así como una colaboración estrecha entre los sectores de salud animal y la salud humana para una gestión integrada en caso de mordidas.

Palabras clave: perro, animales carnívoros, rabia, mordeduras, zoonosis, actitudes, población urbana, Burkina Faso 